FACTORES DE CAMBIO EN LAS ORGANIZACIONES DE LA REGIÓN DE CUAUHTÉMOC, CHIHUAHUA, ANTE LA GLOBALIZACIÓN.

\title{
FACTORES DE CAMBIO EN LAS ORGANIZACIONES DE LA REGIÓN DE
} CUAUHTÉMOC, CHIHUAHUA, ANTE LA GLOBALIZACIÓN

\section{FACTORS OF CHANGE IN THE ORGANIZATIONS OF THE REGION OF CUAUHTÉMOC, CHIHUAHUA, FACING THE GLOBALIZATION}

\author{
Ema Cristina Gutiérrez Enríquez*, Karla Isabel Gutiérrez Enríquez**,

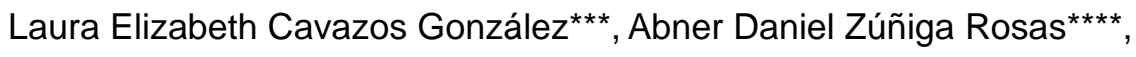

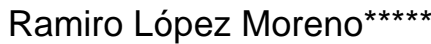

* Maestra en Ciencias en Psicología Social de las Organizaciones. Tecnológico Nacional de México. ORCID: http://orcid.org/0000-0001-8639-7085.

** Ingeniera Industrial. Instituto Tecnológico de Ciudad Cuauhtémoc, Departamento de Ciencias Económico-Administrativas, Cuauhtémoc, Chihuahua. ORCID: http://orcid.org/0000-0002-7515-144X.

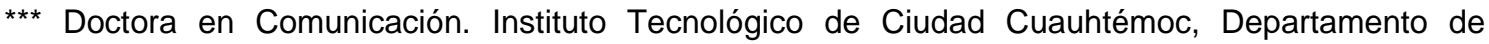
Ciencias Económico-Administrativas, Cuauhtémoc, Chihuahua. ORCID: http://orcid.org/0000-00030705-9876.

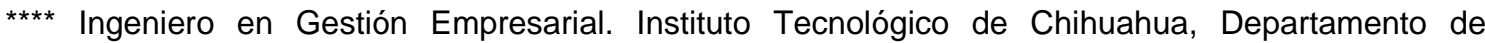
Ingeniería industrial, Chihuahua, Chihuahua. ORCID: http://orcid.org/0000-0002-6283-7370.

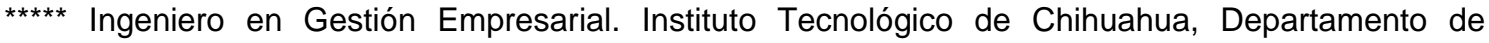
Ingeniería industrial, Chihuahua, Chihuahua. ORCID: http://orcid.org/0000-0002-6171-5769.

Dirección para recibir correspondencia: cristigutie@gmail.com

Fecha de recibido: 30 de octubre de 2018

Fecha de aceptación: 18 de noviembre de 2018

DOI: https://doi.org/10.19136/hitos.a25n71.3230

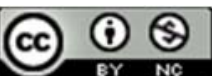

GUTIÉRREZ-enRíQUeZ E. C., GUTIÉRREZ-ENRÍQUEZ K. I., CAVAZOS-GONZÁLEZ L. E., ZÚÑIGA-ROSAS A. D., LÓPEZ-MORENO R. 
FACTORES DE CAMBIO EN LAS ORGANIZACIONES DE LA REGIÓN DE CUAUHTÉMOC, CHIHUAHUA, ANTE LA GLOBALIZACIÓN.

\section{RESUMEN}

OBJETIVO: Detectar las acciones de cambio generadas ante la globalización en las organizaciones de la región de Cuauhtémoc, Chihuahua.

MATERIAL Y MÉTODO: Se visitaron 30 organizaciones dedicadas a comercializar productos, a proporcionar entretenimientos y a la venta de alimentos, se aplicó a los empresarios una encuesta de 30 preguntas de opción múltiple.

RESULTADOS: Se encontró que la mayoría de las empresas visitadas son pequeñas empresas, dirigidas en su mayoría por empresarios jóvenes, que promueven el trabajo en equipo y la participación de los empleados.

CONCLUSIONES: Se concluye que las empresas visitadas están siendo afectadas por la globalización en sus formas de pensar y procesos tanto administrativos como técnicos.

PALABRAS CLAVE: Globalización. Glocalización. Cambio organizacional.

\section{ABSTRACT}

OBJECTIVE: To detect the actions of change generated by the globalization in the organizations of the región of Cuauhémoc, Chihuahua.

MATERIAL AND METHOD: 30 organizations dedicated to market products, provide entertainment, and sell food were visited. A survey of 30 multiple choice questions was applied to the entrepreneurs

RESULTS: It was found that most of the companies that were visited are small businesses, mostly run by young entrepreneurs that promote teamwork and employee participation.

CONCLUSIONS: It is concluded that the companies that were visited are being affected by globalization in their ways of thinking and both administrative and technical processes.

KEY WORDS: Globalization. Glocalization. Organizational change.

GUTIÉRREZ-ENRÍQUEZ E. C., GUTIÉRREZ-enRíQUEZ K. I., CAVAZOS-GONZÁLEZ L. E., ZÚÑIGA-ROSAS A. D., LÓPEZ-MORENO R. 
FACTORES DE CAMBIO EN LAS ORGANIZACIONES DE LA REGIÓN DE CUAUHTÉMOC, CHIHUAHUA, ANTE LA GLOBALIZACIÓN.

\section{INTRODUCCIÓN}

A través de la historia, desde que el hombre era nómada, fue organizándose en grupos para desarrollar las funciones de caza de animales, protección del medio ambiente y poder satisfacer sus necesidades básicas. Con el sedentarismo y la acumulación de bienes, los grupos formaron sociedades, las cuales intercambiaban mercancía a manera de trueque. México, según Rodríguez (1992), ha evolucionado como todas las sociedades, mediante la industrialización que se desarrolló, dando lugar al surgimiento de empresas pequeñas y medianas, con ello se favoreció la generación de empleo nacional y la satisfacción de las necesidades básicas de la población.

Gran parte del desarrollo económico del país, se ha dado por el crecimiento de las empresas pequeñas y medianas que trabajan de manera amplia y actual. Las personas al frente de estas organizaciones, implementan técnicas y estrategias para hacer frente a la problemática interna y del entorno, por lo cual deben tener disposición para el cambio, en la forma de trabajo, de actuar y tomar decisiones. Asimismo, se busca desarrollar elementos que integran la organización con características de actores auto dirigidos.

La región de Cuauhtémoc, Chihuahua, trabaja en un ámbito multicultural, donde predominan dos culturas fuertes, una mestiza y una menonita y en su fuerza laboral incluye a la cultura tarahumara. Esta situación ha ido generando formas de trabajo únicas, incluyentes y complementarias, puesto que cada grupo étnico aporta aspectos importantes para el desarrollo de las empresas. Esta estructura social multicultural, ha dado como resultado una característica propia de la región, sus empresas y sus formas de trabajo, incluyendo el liderazgo empresarial, la estructura de las organizaciones y sus grupos de trabajo, la capacidad de adaptarse al cambio y la resistencia al cambio por parte de las mismas organizaciones y sus integrantes. Además, se observan diferencias en la visión organizacional, en la metodología de trabajo y en las formas de alcanzar los objetivos.

Las condiciones de la región dan lugar a la presente investigación en la cual se realiza un análisis de los cambios organizacionales ocasionados por la influencia de la globalización y la glocalización de las empresas.

GUTIÉRREZ-ENRÍQUEZ E. C., GUTIÉRREZ-enRíQUEZ K. I., CAVAZOS-GONZÁLEZ L. E., ZÚÑIGA-ROSAS A. D., LÓPEZ-MORENO R. 
FACTORES DE CAMBIO EN LAS ORGANIZACIONES DE LA REGIÓN DE CUAUHTÉMOC, CHIHUAHUA, ANTE LA GLOBALIZACIÓN.

\section{MARCO TEÓRICO CONCEPTUAL}

Gutiérrez 2012, cita a Mello F., (1983 y 2004) quién define el cambio como una modificación del comportamiento para realizar exitosamente una actividad o alcanzar un objetivo. Las condiciones sociales, económicas, políticas, y de comercialización a nivel mundial tan cambiantes, obligan a las organizaciones a renovarse en cuanto a estructura, comportamientos, tecnología, métodos, etc. Por lo que deben realizar una revisión periódica de estos aspectos. El autor plantea que existen fuerzas externas e internas que impulsan el cambio organizacional, por lo que deben ser identificadas. Las fuerzas externas son ajenas a la organización, sin embargo, ejercen una influencia, entre ellas están las características demográficas, los avances tecnológicos, cambio en el mercado y las presiones políticas y sociales. Las fuerzas internas son originadas dentro de la organización, y pueden ser un problema, una oportunidad o una necesidad.

Dentro de las fuerzas internas están los empleados con sus capacidades, necesidades, inquietudes, la satisfacción laboral; otra fuerza interna es la forma de dirigir, el trato que se le brinda a los empleados, la oportunidad de participar, la aplicación de la teoría de facultación. La organización debe hacer un balance entre las dos fuerzas para lograr un equilibrio, quizá el problema es de actitud ante la situación. Cuando la organización propone un cambio el personal tiene dos alternativas, lo acepta y lo hace funcionar o lo rechaza y lo hace fracasar. La forma de reaccionar ante un cambio propuesto, varía en forma e intensidad, pero puede convertirse en un grave problema para la organización si no se maneja correctamente. La resistencia al cambio según Kinicki y Kreitner (2003 y 2009), es la respuesta emocional y de conducta que presenta el individuo o el grupo ante la incertidumbre, mientras mayor sea la incertidumbre, mayor será la resistencia, (incertidumbre es la percepción real o supuestas de una amenaza o peligro, en este caso el desconocimiento del cambio), cómo, cuándo y en qué forma reaccionarán, es impredecible, pero puede ser de manera sencilla hasta un sabotaje o paro de labores.

González (2012), argumenta que desde su aparición en los años 80’s, la globalización ha sido generadora de controversias, algunos autores trabajan en favor de ella y otros la rechazan totalmente, se observa que la globalización ha desarrollado desde grandes beneficios hasta provocar pobreza extrema en algunos sectores de la población. Debido a ello, es necesario capacitar profesionalmente a los jóvenes para que puedan hacer frente a las cuestiones de diversidad cultural y pluralidad en las formas de realizar los trabajos. Ante esto las universidades e instituciones educadoras se ven forzadas a actualizar sus planes de estudio acordes a un plan global que sea reconocido a nivel internacional. Para el autor "el GUTIÉRREZ-ENRÍQUEZ E. C., GUTIÉRREZ-ENRíQUEZ K. I., CAVAZOS-GONZÁLEZ L. E., ZÚÑIGA-ROSAS A. D., LÓPEZ-MORENO R. 
FACTORES DE CAMBIO EN LAS ORGANIZACIONES DE LA REGIÓN DE CUAUHTÉMOC, CHIHUAHUA, ANTE LA GLOBALIZACIÓN.

paisaje de una oficina anteriormente estaba lleno de máquinas de escribir y ahora hay computadoras. Ello, demanda nuevas tareas y empleos que hace que se deba dominar el uso de ordenadores". Los trabajadores deben saber leer, manejar la computadora y otro idioma, o se corre el riesgo de ser analfabeto computacional

González (2012), comenta que de esta manera, la globalización tiende a modificar la estructura laboral y las habilidades. Pasando del trabajo individualizado y estático, al trabajo flexible. De ese trabajo de documentos a aquel que tiene que ver con la computadora. Del trabajo dependiente y poco creativo, al independiente. Del trabajo rígido al moldeable, en donde se necesitan distintas capacidades para que los colaboradores puedan conocer y aplicarse a todas las funciones de la empresa. Asimismo, el autor argumenta: "Lógicamente el buen manejo del idioma materno y el conocimiento de algunas otras lenguas para las negociaciones o bien el entendimiento cultural. Estos aspectos son importantes, ya que el trabajador ha hecho que su vida laboral sea más amena". Esto provoca que aumente la demanda de profesionistas con mayores conocimientos y destrezas afines a la actual situación tecnológica.

La globalización ha eliminado de cierta forma las fronteras geográficas entre los países, Arámbula (2003), comenta que la globalización tiene un impacto determinante en la sociedad, en su cultura, en su modo de vida, su forma de hacer negocios, a tal grado que la misma ha tenido apoyo y rechazo de gran parte de la humanidad. Quienes la aceptan ven oportunidades de negocio, de expansión, dominio, crecimiento y riqueza. Quienes la rechazan ven pérdida de valores, de cultura, de identidad nacional, y la amenaza de competir en desventaja con las grandes potencias.

Esta situación obliga a las organizaciones e individuos a cambiar y adaptarse rápidamente ante estas exigencias sociales y económicas, si no lo hacen se quedan en el camino. Las organizaciones tienden a hacerse esbeltas en sus procesos, rápidas y enfocadas a clientes y productos, así como innovadoras, con la idea de subsistir en el siglo XXI. La globalización no tiene vuelta atrás y el éxito de las empresas y organizaciones dependerá de su capacidad de adaptarse al cambio, de innovar y procesar la información. El autor argumenta con relación a la conservación de la cultura en cada país y región frente la influencia de un mundo globalizado, que cada uno de ellos decidirá si mantiene sus raíces o se globaliza. Ante esto las compañías "se están preparando para enfrentar la globalización con éxito; una de las estrategias adoptadas es la de fortalecer la cultura organizacional. En estas empresas, la cultura regula el comportamiento hacia el éxito".

GUTIÉRREZ-ENRÍQUEZ E. C., GUTIÉRREZ-enRíQUEZ K. I., CAVAZOS-GONZÁLEZ L. E., ZÚÑIGA-ROSAS A. D., LÓPEZ-MORENO R. 
FACTORES DE CAMBIO EN LAS ORGANIZACIONES DE LA REGIÓN DE CUAUHTÉMOC, CHIHUAHUA, ANTE LA GLOBALIZACIÓN.

Para Arámbula (2003), la aplicación del comportamiento organizacional al manejo y entendimiento del personal en la organización, son determinantes en el poder adaptarse al cambio y a la globalización, ya que solo se logrará mediante la aceptación y participación de los empleados. Esto implica un gran reto organizacional, señala Mora (2008), citado por Gutiérrez (2015), ya que las organizaciones deben adaptarse a personas que son diferentes entre sí, creando una diversidad laboral, lo cual ha hecho a las organizaciones heterogéneas al contener entre su personal la procedencia de diferentes razas, lugares de origen, ideologías, creencias, culturas; obligando a la organización a entenderlos y guiarlos hacia el objetivo principal, sin perder de vista el bienestar del personal. Los administradores tienen que cambiar su filosofía de tratar a todos por igual y reconocer las diferentes capacidades de las personas. Gutiérrez (2015), cita a Mora (2008), quién comenta que las organizaciones ya no se ven afectadas por las fronteras geográficas de cada país, sino que la globalización convirtió al mundo en un solo lugar, por ello los administradores deben ser capaces de trabajar con personas de diferentes culturas. Asimismo, el administrador cada vez está expuesto a realizar trabajos en el extranjero o con personas extranjeras, para lo cual deberá de comprender sus culturas y adaptarse a esas diferencias.

Alvarado, (2017) argumenta que las organizaciones exitosas deben fomentar la innovación y dominar el arte del cambio o se pondrán en peligro de extinción. El éxito lo alcanzarán las organizaciones que mantengan su flexibilidad, mejoren constantemente la calidad y venzan a la competencia del mercado con una corriente continua de productos y servicios innovadores.

Señala Barba (2000), que se puede considerar a la globalización como los procesos mediante los cuales las corporaciones multinacionales mantienen un nivel de competitividad en los mercados globales, lo cual logran compaginando sus procesos productivos, los de comercialización y de investigación y desarrollo a nivel mundial. Este mundo globalizado incluye algunos países y sectores poblacionales, pero a la vez excluye algunos sectores geográficos y poblaciones marginadas. Al considerar la globalización desde el punto de vista cultural, surge un nuevo concepto y el autor cita a Beck (1988), quién lo propone como glocalización, en lugar de globalización, considerando que en realidad lo que se está dando a nivel mercado, es un acercamiento de las culturas locales y Beck (1998), citado por Barba (2000), argumenta que "lo global y lo local no son excluyentes, ya que lo local debe entenderse como un aspecto de lo global". Gaynor (2005), menciona que las empresas mexicanas deben hacer algo más que sobrevivir a las exigencias del entorno, argumentando que existen algunas particularidades que es conveniente para los empresarios llevar a cabo, en primer lugar, las grandes corporaciones tienen accionistas, a diferencia de las GUTIÉRREZ-ENRÍQUEZ E. C., GUTIÉRREZ-ENRÍQUEZ K. I., CAVAZOS-GONZÁLEZ L. E., ZÚÑIGA-ROSAS A. D., LÓPEZ-MORENO R. 
FACTORES DE CAMBIO EN LAS ORGANIZACIONES DE LA REGIÓN DE CUAUHTÉMOC, CHIHUAHUA, ANTE LA GLOBALIZACIÓN.

organizaciones mexicanas, que cuentan con propietarios, los cuales toman todas las decisiones de manera centralizada.

Además, las grandes corporaciones manejan un gran apego a los grupos de interés (stakeholders), en la organización como los accionistas, trabajadores, proveedores, clientes, comunidad que se ven afectados con las decisiones organizacionales. González (2012), comenta que una cuestión muy importante en el impacto de la globalización ante las organizaciones, es el papel de la educación de los profesionistas, la cual debe ir a la par de las exigencias de la globalización. El autor comienza explicando que a la globalización se le han dado diferentes definiciones y enfoques, por un lado, se le ve "como un proceso que tiene que ver con la economía, el arte, la cultura, la religión, etcétera". De igual forma se ha definido como "globalismo" a aquella globalización en donde predomina la generación de utilidades, las inversiones, el comercio y todo lo relacionado a la parte económica. A esta definición se le agrega la globalidad que tiene que ver con la época moderna o bien con el desarrollo del capitalismo, según Beck (1994), citado por González (2012).

Una meta a alcanzar por la mayoría de las organizaciones, es establecer en su estructura una forma de comportamiento organizacional, que facilite los procesos y el logro de los objetivos planteados. Para ello, se debe de fomentar una cultura organizacional que favorezca lo propuesto argumenta Gross (2010), ya que mediante los patrones de conducta que se fomenten, será más sencillo cumplir con el cometido o más difícil lograrlo. Por años las organizaciones y sus administradores han tratado de predecir los comportamientos de los empleados, implementando técnicas de selección de personal que les permitan de alguna forma predecir los desempeños futuros en la organización. De ahí, la importancia de fomentar una cultura de desempeño con calidad, enfocada al servicio o producción, pero que determine en el trabajador una entrega total al logro de los objetivos organizacionales.

El autor menciona cuatro modelos de comportamiento organizacional, los cuales son tradicionales en su aplicación en las organizaciones, estos modelos enmarcan las formas de los administradores de establecer una forma generalizada en la organización para hacer las cosas y alcanzar los objetivos. Entre los modelos del comportamiento organizacional, está el de Custodia, en el cual el administrador, jefe o gerente se da cuenta del sentimiento que los empleados tienen ante la satisfacción o insatisfacción que les causa el trabajo realizado y el ambiente en el que lo realizan, enfocándose a brindar un ambiente de satisfacción laboral. De igual forma, el gerente o administrador mediante el modelo de Apoyo, trata de dar al trabajador un soporte para su desarrollo personal con la idea de que el trabajador se comprometa con la organización. En el modelo Colegial, la administración trata de dar la GUTIÉRREZ-ENRÍQUEZ E. C., GUTIÉRREZ-ENRÍQUEZ K. I., CAVAZOS-GONZÁLEZ L. E., ZÚÑIGA-ROSAS A. D., LÓPEZ-MORENO R. 
FACTORES DE CAMBIO EN LAS ORGANIZACIONES DE LA REGIÓN DE CUAUHTÉMOC, CHIHUAHUA, ANTE LA GLOBALIZACIÓN.

sensación de que se trabaja en un grupo colegiado, enfocado al logro de los objetivos, se basa en el principio de camaradería, mediante el cual se supone que el trabajador se siente parte importante del equipo de trabajo y se pone la camiseta. El modelo más antiguo del comportamiento organizacional es el autocrático, basado en la formalidad de la autoridad, es inflexible y se enfoca en controlar el comportamiento de los demás, esperando que los trabajadores obedezcan y sigan órdenes.

Según Cano (2006), el cambio organizacional se puede entender "como una modificación del estado de cosas en algo que nos atañe o importa; o una transición en nuestro status quo, para mejorar o empeorar". El autor cita a Kauffman, (1997), quien argumenta que el proceso de cambio "es el medio de que dispone una organización para transformarse y aplicar una nueva visión". Asimismo, el autor menciona que si las organizaciones están interesadas en mantenerse en un mercado competitivo, deberán adaptarse rápidamente a las demandas del entorno económico, político y social. Señalando que el entorno incluye clientes, proveedores, comunidad, entre otros, y que debe abarcarlos todos para asegurar el éxito. Los cambios drásticos tecnológicos obligan a las organizaciones a cambiar sus perspectivas y estrategias empresariales. El autor argumenta que "los países, y sobre todo los subdesarrollados deben enfrentar la radical transformación que se está produciendo en las condiciones de competitividad y de inserción en la economía mundial, desarrollando organizaciones y programas que impliquen nuevas estrategias de mercado". Por tanto, "el cambio debe darse en la cultura empresarial, en donde las condiciones de los mercados son las que marcan la pauta para incorporar nuevas tendencias en la dirección y administración de las organizaciones, tarea que, de no hacerse, implica una gran probabilidad de desaparecer". Todas las organizaciones privadas y públicas están obligadas a cambiar permanentemente sus estructuras, objetivos y estrategias, de tal forma que puedan estar preparadas para enfrentar las nuevas exigencias de la globalización, llegando a ser de clase mundial.

La región de Cuauhtémoc, Chihuahua, ha crecido en el área de empresarial de una manera sorprendente, ya que actualmente se establecieron cadenas comerciales que a nivel mundial son muy reconocidas (Wal-Mart, Sam's, etc.), creando con esto un concepto de crecimiento en la economía de la ciudad puesto que este tipo de empresas no se establecen si la situación económica de la ciudad no es favorable o es baja su capacidad de generar que la economía se mueva o crezca.

La globalización está alcanzando la región y se están rompiendo barreras de cultura e idiomas, dejando atrás los paradigmas y las formas de hacer las cosas, considerando que GUTIÉRREZ-ENRÍQUEZ E. C., GUTIÉRREZ-ENRÍQUEZ K. I., CAVAZOS-GONZÁLEZ L. E., ZÚÑIGA-ROSAS A. D., LÓPEZ-MORENO R. 
FACTORES DE CAMBIO EN LAS ORGANIZACIONES DE LA REGIÓN DE CUAUHTÉMOC, CHIHUAHUA, ANTE LA GLOBALIZACIÓN.

no sólo se puede ser competente a nivel ciudad o Estado, si no, ir a niveles internacionales y para un futuro, mundiales. Esto, convierte a la región de Cuauhtémoc en una ciudad en crecimiento económico y a nivel empresarial en un mercado más globalizado, que se adapta a las exigencias del mercado mundial.

\section{MATERIAL Y MÉTODO}

El proyecto se realizó en la región de ciudad Cuauhtémoc, considerando para el análisis 30 empresas locales de servicios, dedicadas al comercio, entretenimiento y venta de alimentos, las cuales fueron seleccionadas a conveniencia, considerando aquellas que accedieran a contestar la encuesta. Todas ellas de tamaño pequeño.

La investigación se caracteriza por ser cuantitativa y se realizó un análisis descriptivo.

El objetivo es detectar las acciones de cambio organizacional, ante la globalización en las organizaciones de la región de Cuauhtémoc. El instrumento de medición, que se utilizó fue una encuesta compuesta de 30 preguntas tipo opción múltiple. La encuesta se realizó con los empresarios que accedieron a contestar.

\section{RESULTADOS}

Mediante el análisis de los datos, se encontró que el 69\% de los empresarios encuestados se encuentra en un rango de 30 a 50 años de edad, el 83\% son casados, el $70 \%$ de ellos cuenta con licenciatura y el $20 \%$ con maestría, el $70 \%$ son del género masculino.

En relación a la empresa, el 70\% manifiesta que cuentan con un organigrama formal, el 92\% tiene menos de 100 empleados, de los cueles solo el 29\% tiene estudios profesionales.

El 67\% de los empresarios permite que sus empleados tengan participación en toma de decisión en cuanto a las mejoras de la empresa y en el planteamiento de objetivos, el $40 \%$ de los colaboradores se involucra en establecer tiempos de trabajo y al $30 \%$ se les delega autoridad y toma de decisión. El trabajo en equipo es promovido por el $93 \%$ de las empresas, y se percibe que el $93 \%$ de los empleados tienen compromiso con la organización.

El 60\% de los empresarios asisten a eventos de actualización, al menos una vez al año, ya sean congresos, expos o cursos, los cambios en la organización son promovidos una o dos veces al año por el $89 \%$ de los empresarios, en las áreas de ventas y procesos

GUTIÉRREZ-ENRÍQUEZ E. C., GUTIÉRREZ-enRíQUEZ K. I., CAVAZOS-GONZÁLEZ L. E., ZÚÑIGA-ROSAS A. D., LÓPEZ-MORENO R. 
FACTORES DE CAMBIO EN LAS ORGANIZACIONES DE LA REGIÓN DE CUAUHTÉMOC, CHIHUAHUA, ANTE LA GLOBALIZACIÓN.

administrativos el $97 \%$ y en mercadotecnia el $60 \%$, los procesos productivos son modificados solo por el $30 \%$ de ellos.

El uso de la tecnología en el área administrativa se emplea principalmente en medidas de control del personal, en el área de producción en la ingeniería de procesos, en el área de ventas el uso que se le da es a través de Internet, aplicaciones, cajas de venta y promoción del producto. El 50\% informa que su equipo tecnológico está actualizado en un 50\%, únicamente.

El 53\% de los empresarios, informan que realizan análisis del comportamiento del mercado y están al tanto de los cambios en el entorno, así como cambios sociales, económicos y políticos. El mercado al que se enfoca la empresa es en un $80 \%$ regional y estatal, solo el $20 \%$ abarca un mercado nacional. Sólo el $17 \%$ utiliza la publicidad por Internet, el 53\% cuenta con página Web del negocio. Únicamente el 50\% de los negocios aceptan pagos con tarjeta o transferencia bancaria.

El 53\% de los negocios busca franquiciarse, maneja el inventario con código de barras y emite facturación electrónica, los proveedores son en un $70 \%$ estatales y regionales, el $20 \%$ nacionales y el $10 \%$ internacionales. El $53 \%$ busca estar certificado en las normas ISO y consideran a la globalización como un agente de cambio.

\section{CONCLUSIONES}

Con la información analizada, se puede llegar a una conclusión clara de cuál es el impacto que tiene la globalización en ciudad Cuauhtémoc.

La interpretación de resultados es bastante extensa; sin embargo, se pueden hacer varias afirmaciones y conclusiones respecto a la investigación realizada, tales como:

La globalización está afectando directamente en la forma de llevar a cabo los controles financieros, un claro ejemplo es la facturación electrónica, que cada vez se obliga a tener en la empresa y si no se cuenta con un sistema adecuado, tarde o temprano la empresa desaparecerá del mercado.

Cada vez son más las organizaciones que se suman a que sus empleados tengan voz y voto en los procesos de mejora y son tomados en cuenta para combatir problemas que se presentan en las empresas.

GUTIÉRREZ-ENRÍQUEZ E. C., GUTIÉRREZ-enRíQUEZ K. I., CAVAZOS-GONZÁLEZ L. E., ZÚÑIGA-ROSAS A. D., LÓPEZ-MORENO R. 
FACTORES DE CAMBIO EN LAS ORGANIZACIONES DE LA REGIÓN DE CUAUHTÉMOC, CHIHUAHUA, ANTE LA GLOBALIZACIÓN.

También mencionar, que se están abriendo panoramas más elevados hacia un crecimiento del mercado en la ciudad de Cuauhtémoc, ya que se abren puertas a nuevos proveedores y equipo tecnológico para las diferentes áreas y más aún en producción.

En cuanto a los líderes de las empresas, la mayor parte son jóvenes de 30 a 50 años con licenciatura, lo que quiere decir, que este sistema globalizado ha tenido mucho que ver para que los militantes de altos puestos estén cada vez más preparados y hoy en día crece la cantidad de trabajadores que se prepara en materia de educación. También destacar el papel que tienen las mujeres en los altos mandos jerárquicos de las organizaciones, lo cual han roto barreras contra la mujer, y hoy, con la globalización, cualquiera tiene acceso a la información y puede aspirar a tener grandes metas con el uso adecuado de la información.

En ciudad Cuauhtémoc, Chihuahua, las empresas han evolucionado poco a poco y son algunas las que se reúsan a cambiar sus métodos; sin embargo, la globalización es una ola mundial que empuja a todos hacia un mismo orden, hacia un nuevo mercado y sea de una forma u otra, las empresas están cambiando, el mercado está cambiando, la gente está cambiando, así que los que se nieguen a innovar, actualizarse y a alinearse al nuevo modelo, podrían desaparecer del ámbito comercial.

En general, se puede decir que la globalización afecta a las empresas, pero un punto a favor de la ciudad de Cuauhtémoc, es que la cultura empresarial también va cambiando y muchos empresarios tienen visión e innovación y buscan talentos y herramientas que puedan ayudarles a cumplir su meta, también existen organizaciones de asesoría, cursos, talleres y pláticas a líderes empresariales para estar al día en las nuevas tendencias y actualizaciones.

Además, las formas de emprender en la región de Cuauhtémoc, han logrado establecer una glocalización en sus procesos y les da características distintivas de otras regiones del Estado.

GUTIÉRREZ-ENRÍQUEZ E. C., GUTIÉRREZ-enRíQUEZ K. I., CAVAZOS-GONZÁLEZ L. E., ZÚÑIGA-ROSAS A. D., LÓPEZ-MORENO R. 
FACTORES DE CAMBIO EN LAS ORGANIZACIONES DE LA REGIÓN DE CUAUHTÉMOC, CHIHUAHUA, ANTE LA GLOBALIZACIÓN.

\section{REFERENCIAS BIBLIOGRÁFICAS}

Arámbula, M. (2003). El impacto de la globalización en la formación de una cultura organizacional. Recuperado de http://207.249.140.110/cgi-bin/contenido.pl?Art=286

Barba, A. (2000). Cambio organizacional y cambio en los paradigmas de la administración, Iztapalapa. Revista de Ciencias Sociales y Humanidades, 22(48) Recuperado de http://148.206.53.230/revistasuam/iztapalapa/include/getdoc. php?id=648\&article=659\& mode=pdf

Cano, M. (2006). El liderazgo transformacional: sustento de las organizaciones de clase mundial, Revista Ciencia Administrativa, 17(2). Recuperado de http://www.uv.mx/iiesca/revista/documents/liderazgo2006-2.pdf

Gaynor, E. (2005). ¿Qué pueden hacer las organizaciones en la era de globalización?, The Organization Development Institute International, Latinoamérica, Argentina. Recuperado de http://www.gestiopolis.com/canales5/ger/desaquepued.htm

González, J., (2012). La globalización en el siglo XXI y su impacto en la educación, la cultura y las habilidades, Contribuciones a la Economía, (enero 2012). Recuperado de http://www.eumed.net/ce/2011b/

Gross, M. (2010). Cuatro modelos de comportamiento organizacional, pensamiento imaginativo. Recuperado de http://manuelgross.bligoo.com/content/view /780384/Cuatro-Modelos-de-Comportamiento-Organizacional.html

Gutiérrez, E., (2012). Comportamiento organizacional. Recuperado de http://www.lulu.com/shop/ema-cristina-guti\%C3\%A9rrez-enr\%C3\%ADquez /comportamiento-organizacional/hardcover/product-22425111.html

Gutiérrez, E. (2015). Comportamiento Organizacional (2a ed.). México: Instituto Tecnológico de Cd. Cuauhtémoc.

Kinicki, A. y Kreitner, R. (2009 y 2012). Organizational behavior. México: McGraw-Hill.

Rodríguez, J. (1992). Como administrar pequeñas y medianas empresas. México: ECASA.

GUTIÉRREZ-ENRíQueZ E. C., GUTIÉRREZ-ENRíQUEZ K. I., CAVAZOS-GONZÁLEZ L. E., ZÚÑIGA-ROSAS A. D., LÓPEZ-MORENO R. 